\title{
Locus of inhibition effects in the priming of lexical decisions: pre- or postlexical access?
}

\author{
ROBERT F. LORCH, JR. \\ University of Kentucky, Lexington, Kentucky \\ DAVID A. BALOTA \\ Washington University, St. Louis, Missouri \\ and \\ EDWARD G. STAMM \\ University of Kentucky, Lexington, Kentucky
}

\begin{abstract}
The lexical decision task has been employed to investigate the effects of semantic context on word recognition. A frequent finding from the task is that "word" responses are slower when the target is preceded by an unrelated word than when it is preceded by a neutral stimulus. This inhibition effect has been interpreted as indicating that the unrelated prime interferes with wordrecognition processes operating on the target. In three experiments, the effects of unrelated primes were compared for a lexical decision and word naming task. Although large inhibition effects were found for the lexical decision task in all experiments, no inhibition effects were observed for the naming task. The results are interpreted as demonstrating that inhibition effects in the lexical decision task are not on recognition processes; rather they are located at processes operating after recognition of the target has occurred.
\end{abstract}

The priming paradigm has been used in conjunction with the lexical decision task to study such diverse topics as attention (Neely, 1977), word recognition (Becker, 1980), and memory retrieval (Meyer \& Schvaneveldt, 1971). One important finding from this research is that subjects are often slower to respond to a target word if the preceding prime is unrelated to the target than if the prime is neutral (Becker, 1980; Neely, 1976). This inhibition effect has often been interpreted as indicating that processing of an unrelated prime delays word-recognition processes (Becker, 1980; Neely, 1977). An alternative possibility, however, is that the inhibition is due to effects of the prime-target relation on processes occurring after a lexical representation of the target word has been accessed. The current investigation concerns the locus of inhibition effects in the lexical decision task. In the following sections, we will elaborate on the theoretical relevance of this issue and present a strategy for resolving the question.

\section{Context Effects on Word Recognition}

The issue of the locus of inhibition effects in lexical decision is directly relevant to both Becker's (1980) verification model of word recognition and Posner and Snyder's

We thank Janet Duchek, Alice Healy, and an anonymous reviewer for their helpful comments on an earlier draft of this manuscript. Requests for reprints should be addressed to: R. F. Lorch, Jr., Department of Psychology, University of Kentucky, Lexington, KY 40506-0044. (1975a, 1975b) two-process model. However, because the current experiments were designed specifically to test the verification model, the implications for two-process theory will be considered only in the Discussion.

Becker proposed that when a word is presented without context in a word recognition task, a "sensory set" consisting of possible lexical interpretations of the target item is constructed based upon the visual features of the stimulus. The items in the sensory set are then compared with a visual representation of the target in a frequencyordered, self-terminating comparison process. When a match is determined, a "word" response is given; if no match is found, a "nonword" response is given. If a word is preceded by a context word, however, a different sequence of processes occurs. When a word prime is presented, associates of the prime are entered into a "semantic set." When the target is subsequently presented, the items in the semantic set are compared with the stimulus during the time that the sensory set is being established. If an item in the semantic set matches the target, a "word" response is given; if no match is found, the process of comparing the target with the items in the sensory set is then initiated.

According to the verification model, the critical determinant of the pattern of facilitation and inhibition effects found in a lexical decision task is the size of the semantic set generated on word prime trials. If the set size is small, then substantial facilitation will result on related prime trials and little or no inhibition will result on unrelated prime trials (i.e., facilitation dominance). This is because 
the semantic set can be compared with the target before the sensory set is even established. On the other hand, there will be less facilitation on related trials when the semantic set is large because it will take longer to find a match during the semantic set comparison. In addition, there will be large inhibition effects on unrelated prime trials because the process of comparing the semantic set will delay the necessary stage of comparing the sensory set (i.e., inhibition dominance). According to Becker, the size of the semantic set will depend upon the distribution of prime-target associations across items in an experiment. If the primes and targets are all strong associates, then the semantic set will be small and facilitation dominance should result. If the prime-target associations vary widely in strength, then the semantic set will be large and inhibition dominance will result. Becker (1980) reported findings supporting these predictions: Facilitation dominance is found in a lexical decision task when related prime-target pairs are consistently strongly associated; inhibition dominance is found when category-exemplar pairs representing a wide range of strength of association are used as stimuli.

Becker's (1980) verification model attributes inhibition effects to processing that occurs before presentation of the target. The semantic set is established in the interval between prime and target presentation, and the size of the set determines the pattern of priming effects. If inhibition effects are demonstrated to occur after lexical access, then Becker's model must be modified.

\section{A Strategy for Localizing Inhibition Effects}

There is good reason to suspect that inhibition effects in the lexical decision task may reflect postlexical access processes (de Groot, 1983; de Groot, Thomassen, \& Hudson, 1982). Given the binary decision that subjects are required to make in the task, any variable that is correlated with the lexical status of the target item becomes a potential candidate for affecting the decision process (Balota \& Lorch, in press; Seidenberg, Waters, Sanders, \& Langer, 1984; West \& Stanovich, 1982). In fact, there is substantial evidence that subjects do construct decisionmaking strategies based upon confoundings of variables with the word-nonword response (Chumbley \& Balota, 1984). For example, there is evidence that subjects base lexical decisions on judgments of the frequency of usage of the target item in the English language (Balota \& Chumbley, 1984). More important in the present context, several experiments have demonstrated that many priming effects observed in a lexical decision task do not occur in a naming task (Lupker, 1984; Seidenberg et al., 1984; West \& Stanovich, 1982). On the assumption that the lexical decision and naming tasks differ primarily in the postlexical processes they involve, these findings have been interpreted as indicating that postlexical processes play an important role in priming in the lexical decision task. However, only the West and Stanovich study included a neutral baseline to allow separation of facilitation and inhibition effects when comparing priming in the lexical decision and naming tasks. Although they found inhibition effects only in lexical decision, they employed sentence contexts as primes rather than single-word contexts. As they note. the inhibition effects which they found for lexical decision may have been due to sentence integration processes occurring subsequent to word recognition, and thus may not generalize to single-word priming situations.

The current investigation compares single-word priming in lexical decision and naming to determine whether inhibition effects in lexical decision are pre- or postlexical (Balota \& Chumbley, 1984; Chumbley \& Balota, 1984; Lupker, 1984; Seidenberg et al., 1984; West \& Stanovich, 1982). The rationale for this approach is twofold. First, the naming task does not involve a binary decision, so there is no decision process to bias. More generally, the naming task is assumed to minimize postlexical processing relative to the lexical decision task (Forster, 1979; Theios \& Muise, 1977). Second, the verification model is a general model of word recognition, so tests of the theory should not be limited to the lexical decision task. On this reasoning, any priming effects that are observed in both tasks are attributed to prelexical processes; any priming effects that are found only in lexical decision are attributed to postlexical processes.

The experiments reported here were designed to establish favorable conditions for the observation of inhibition based upon results from lexical decision experiments. The design of each experiment was essentially the same. Each experiment compared a lexical decision task and a naming task. Within an experiment, the prime-target word pairs were identical for both tasks; across the experiments, the prime-target pairs were varied. The prime-target relation was manipulated in the same manner in each experiment: the prime was either related, unrelated, or neutral with respect to the target word. The experimental procedures were designed to produce large inhibition effects based on previous findings. First, subjects were explicitly instructed to expect that the prime and target would be related. Second, a high proportion of the word trials involved related prime-target pairs: the proportion of related trials was .66; the proportion of unrelated trials was .17; and the proportion of neutral trials was .17 . On word trials, the conditional probability of a related target given a nonneutral word prime was therefore .8. Finally, the interval between prime and target presentation was sufficiently long to permit conscious expectations to develop ( $\mathrm{SOA}=1,450 \mathrm{msec}$ ). Inhibition effects have consistently been observed under similar conditions in previous lexical decision experiments (den Heyer, Briand, \& Dannenbring, 1983; Neely, 1977).

It was assumed that inhibition effects would be observed in the lexical decision task of each experiment. According to the verification model, similar inhibition (and facilitation) effects should be observed in naming. This is because the priming events are identical for the lexical decision and naming tasks, and the model attributes priming effects to subjects' responses to the prime (i.e., to pre- 
lexical processes). If inhibition is due to postlexical effects of the prime, however, then a different pattern of results is predicted. On the assumption that the naming task involves minimal postlexical processing, no inhibition should be observed in the naming condition of any of the experiments. Note that similar patterns of facilitation might be expected for lexical decision and naming given previous evidence that the locus of facilitation effects is often prelexical (Becker \& Killion, 1977; West \& Stanovich, 1982).

\section{EXPERIMENT 1}

\section{Method}

Subjects. Subjects were undergraduate students at the University of Kentucky who participated in partial fulfillment of a course requirement. Excluding 11 subjects who made too many errors, 24 subjects were assigned to the lexical decision task and 48 subjects were assigned to the naming task. Of the 11 subjects whose data were discarded, 4 were in the lexcial decision condition and 7 were in the naming condition.

Apparatus. All stimulus presentation and data collection were controlled by an Apple II+ microcomputer which was interfaced with a Zenith data systems video monitor. A Mountain Hardware timing board was used to obtain reaction times to the nearest millisecond. A Lafayette model $6602 \mathrm{~A}$ voice-key was interfaced with the computer to record responses in the naming task.

Materials. The critical stimuli were based upon categoryexemplar word pairs selected from the Battig and Montague (1969) norms. Two different sets of items were used. For each set, 12 exemplars were selected from each of 5 semantic categories. Across sets, the mean dominance value of the exemplars was 175 (39\%). The two sets of items were based on different semantic categories (Color, Clothing, Metal, Animal, and Tree were the categories in one set; Fish, Bird, Fruit, Furniture, and Cloth were used in the other set). The 60 items in each set were divided into two blocks, such that each of the five categories was represented six times in each block. Within each block, 20 related prime pairs were formed by using the first word of each associated pair as the prime and the second word as the target (e.g., bird-robin). An additional 5 neutral prime pairs were constructed by replacing the first word of the original pair with the word blank (see de Groot et al., 1982). The other 5 items in each block were unrelated prime pairs. Unrelated pairs were constructed by replacing the first word of the original word pair with one of the other four category names (e.g., fruit-eagle). Six different lists of 60 pairs each were generated for each set in order to counterbalance the assignment of targets to priming conditions. Across the lists, each target was paired four times with a related prime and one time each with a neutral and unrelated prime. A target word occurred only once in a given list.

The nonword items for the lexical decision task were generated by selecting exemplars from categories not used for the word trials, and changing one, two, or three letters to create a pronounceable nonword. The category labels used on word trials were paired with nonwords to generate word prime trials for the nonwords. Each category label occurred equally often on word and nonword trials within each block of the lexical decision condition. Within each block, 25 nonword targets were each paired with a category prime and 5 nonword targets were each paired with the neutral prime. As was the case for word pairs, the assignment of target nonwords to priming conditions was counterbalanced across the six stimulus lists. All target words and nonwords were between three and eight letters long and were either one or two syllables.

In addition to the critical items for the experimental blocks, practice items and buffer items were constructed. Two buffer items were included at the start of each block. These buffer pairs were always related word pairs in both tasks. A block of practice items was generated for the purpose of familiarizing subjects with the procedure before presenting the experimental blocks. For the naming task, 30 category-exemplar word pairs were used to construct the practice block. The practice block had the same characteristics as the experimental blocks in the naming task. For the lexical decision task, 12 word pairs and 12 nonword pairs were generated from the 30 items used in the naming task. (The additional 6 items were included in the naming condition because subjects typically required more experience with the response apparatus in that condition before they were able to respond consistently.) These stimuli were used to construct a practice block with the same characteristics as the experimental blocks in the lexical decision task. The categories used in the practice and buffer trials were the same as those used in the experimental blocks. The order of presentation of stimuli within each block was randomized independently for each subject (with the exception of the buffer trials)

Procedure. Each subject participated individually in a session lasting between 20 and $30 \mathrm{~min}$. Subjects were instructed that they would be presented a pair of stimuli on each of many trials. They were told that the first stimulus would be the name of a semantic category on most trials, and the word blank on one sixth of the trials. Subjects were instructed that when the first word was blank, they should get ready for the second stimulus. They were instructed that when a category name was presented first, they should use the first word to prepare for the second stimulus. They were informed that when the first word was the name of a category, the second word would usually be a member of the category. (Subjects in the lexical decision task were told that this would be true only if the second stimulus was a word.) Both speed and accuracy were emphasized in the instructions. The experimenter remained in the lab during the practice block to be certain that the subject fully understood the task.

The sequence of events on each trial was as follows: (1) a row of three asterisks was presented in the center of the video display screen for $200 \mathrm{msec}$; (2) a blank screen was presented for $200 \mathrm{msec}$; (3) a warning tone was presented for $140 \mathrm{msec}$; (4) a blank screen ws presented for $200 \mathrm{msec}$; (5) the prime word was presented for 1,400 msec; (6) a blank screen was presented for $50 \mathrm{msec}$; (7) the target stimulus was presented until a response was made. The response differed for the two tasks. Subjects in the lexical decision task decided whether the second stimulus was a word or nonword. They pressed the " 0 " key to indicate a "word" response and the " 1 " key to indicate a "nonword" response. If the response was correct and the reaction time was less than $1 \mathrm{sec}$, an intertrial interval of $2 \mathrm{sec}$ was initiated. If an error was made or the reaction time exceeded $1 \mathrm{sec}$, an appropriate feedback message was presented (i.e., "Error" or "Your response was longer than 1 second"). Following an error or slow response, the intertrial interval did not begin until the subject pressed a key to begin again. Subjects in the naming task responded to the second word by saying it aloud. After the pronunciation was detected, the following message was presented: "Type ' 0 ' if your response was correct. Type ' 1 ' if there was any problem." Subjects were instructed to type " 1 " if they mispronounced the word, if they triggered the voice key prematurely (e.g., by coughing), or if the voice key failed to detect their response. An intertrial interval of $2 \mathrm{sec}$ began after the subject pressed the "I" or " 0 " key. Unlike the lexical decision condition, error messages were not displayed in the naming task because the self-scoring procedures made subjects aware of their errors. Also, subjects were not informed of response latencies over $1 \mathrm{sec}$ because slow responses were not entirely under their control; rather, some slow responses were due to equipment malfunctions (e.g., failure to detect a vocal response)

\section{Results}

An overall ANOVA was conducted separately on the RTs and errors; then comparisons were carried out to assess the inhibition and facilitation effects separately for the lexical decision and naming tasks. The design of the 
experiment included: the between-subjects factors of task (lexical decision or naming), materials (two different sets of category-exemplar pairs), and list (six levels to counterbalance assignment of items to priming conditions); and the within-subjects factor of prime (related, neutral, or unrelated). The level of significance in all tests is .05 , unless otherwise noted.

For both tasks, RTs exceeding $1 \mathrm{sec}$ were considered errors. In addition, subjects were replaced if they made more than 10 errors on the 60 critical targets in an experiment. It should be noted that it is difficult to interpret error rates in the naming conditions because they represent several different types of errors (mispronunciations; premature triggerings of the voice key; failures to trigger the voice key; slow responses). Despite the variety of ways in which errors could be recorded in naming, the error rates were relatively low and constant across conditions.

Reaction times. The results of the experiment are summarized in Table 1. It may be seen that the pattern of priming effects depended upon the task $[F(2,96)=7.25$, $M S e=900$ for the task $\times$ prime interaction]. Several planned comparisons were conducted to analyze this interaction. The results for the lexical decision task demonstrate inhibition dominance: The 8-msec facilitation effect for the related condition was not reliable $(F<1)$, whereas the $41-\mathrm{msec}$ inhibition effect for the unrelated condition was significant $[F(1,12)=7.11, M S e=2,944]$. Considering the results for the naming task, neither the facilitation effect for the related condition $(F<1)$ nor the inhibition effect for the unrelated condition were reliable $[F(1,36)=2.08, M S e=761]$. The inhibition effect in the lexical-decision task was larger than the inhibition effect in naming $[F(1,48)=6.92, M S e=1,307]$. There was no difference in the magnitude of the facilitation effects $(F<1)$. The only other reliable result was that responses were faster in naming than in lexical decision $[F(1,48)=4.87, M S e=9,654$ for the main effect of task].

Errors. The error rates are also presented in Table 1. The pattern of priming effects on errors differed for the two tasks $[F(2,96)=24.54, M S \mathrm{e}=.0059$ for the task $X$ prime interaction]. Separate tests of the facilitation and inhibition effects for each task demonstrated that only the $13.75 \%$ inhibition effect for the lexical decision task was reliable $[F(1,12)=11.46, M S e=.0198]$. The $3.23 \%$ facilitation effect in lexical decision was marginally significant, however $[F(1,12)=4.55, M S \mathrm{e}=.0028, p=$ .0542].

In summary, Experiment 1 was successful in demonstrating a robust inhibition effect in the lexical decision task. The pattern of inhibition dominance found for lexical decision is consistent with previous findings when category-exemplar pairs have been used as stimuli (Becker, 1980). Despite the large inhibition effect in lexical decision, no inhibition was observed in naming. Thus, the findings suggest that the effect of an unrelated prime is on postlexical processes rather than prelexical processes. Experiment 2 was conducted to provide a further test of this conclusion.

\section{EXPERIMENT 2}

Because all of the target words in Experiment 1 were selected from only five different semantic categories, it was thought that the conditions of the experiment might not be optimal for producing inhibition. Perhaps across trials, subjects learned to prepare in some general fashion for exemplars from any of the categories. Such an effect might reduce the otherwise inhibitory effects of an unrelated prime. Experiment 2 was conducted to eliminate this concern. Stimuli were selected from 30 different categories for use in the experiment. The inclusion of 30 different categories eliminated the possibility of some type of general preparation for target words. A high- and a low-dominance exemplar were selected from each category in order to create variability in the strengths of the prime-target associations on related prime trials. Recall that this is a necessary condition for the observation of inhibition dominance according to Becker (1980). In addition, as described in the Discussion section, the manipulation of dominance permits a further test of the locus of priming effects in the two tasks.

\section{Method}

Subjects. Subjects were undergraduate students at the University of Kentucky who participated in partial fulfillment of a course requirement. After excluding 5 subjects for making too many errors, 24 subjects were assigned to the lexical decision task and 48 subjects were assigned to the naming task. Of the excluded subjects, 2 were from the lexical decision condition and 3 were from the naming condition.

Materials. The critical stimuli were again category-exemplar pairs (Battig \& Montague, 1969; Shapiro \& Palermo, 1970). Unlike exemplars in Experiment 1, however, exemplars were selected from 30 different categories. Two exemplars differing in dominance were selected from each category. Pairs were assigned to two blocks, such that each category was represented once per block and each block contained 15 exemplars at each level of dominance. Once items were assigned to blocks, target words were assigned to the three priming conditions by the same procedures followed in Experiment 1. Again, six lists of stimuli were constructed to counterbalance the assignment of targets to priming conditions.

The generation of nonword items for the experimental blocks followed procedures analogous to those of Experiment 1. The selec-

Table 1

Mean Reaction Times and Percentage Errors (in parentheses) as a Function of Task and Prime for Experiment 1

\begin{tabular}{lccccc}
\hline \multicolumn{1}{c}{ Task } & Related & Neutral & Unrelated & Facilitation & Inhibition \\
\hline Lexical Decision & $545(5.10)$ & $553(8.33)$ & $594(22.08)$ & $8(3.23)$ & $41(13.75)$ \\
Naming & $526(5.47)$ & $532(6.67)$ & $540(5.00)$ & $6(1.20)$ & $8(-1.67)$ \\
\hline
\end{tabular}


tion of buffer items and the construction of the practice block for each task also followed procedures similar to those of Experiment 1.

Procedure. The procedure was the same as in Experiment 1.

\section{Results}

The design of Experiment 2 was similar to that of Experiment 1 except for the additional within-subjects variable of dominance. Also, only one set of stimulus materials was included in Experiment 2.

Reaction times. The findings of the experiment are summarized in Table 2. Averaging over the levels of dominance, it may be seen that the results of Experiment 2 are virtually identical to those of Experiment 1 . As before, priming effects varied over the two tasks $[F(2,120)$ $=9.89, M S e=1,740$ for the task $\times$ prime interaction]. Separate tests of the overall facilitation and inhibition effects demonstrated that only the $47-\mathrm{msec}$ inhibition effect in lexical decision was reliable $[F(1,18)=13.86$, $M S e=2,031]$. The inhibition effect in lexical decision was again larger than the inhibition effect in naming $[F(1,60)=10.24, M S e=2,290]$.

There were several effects of dominance. First, responses were faster to high-dominance targets than to lowdominance targets $[F(1,60)=14.26, M S \mathrm{e}=928$ for the main effect of dominance]. However, the dominance effect occurred in lexical decision, not in naming $[F(1,60)$ $=11.23, M S e=928$ for the task $\times$ dominance interaction; see also Chumbley \& Balota, 1984]. More important are the effects of dominance on priming. Although the prime $X$ dominance interaction was only marginally reliable $[F(2,120)=2.51, M S e=1,778, \mathrm{p}<.09]$, planned comparisons demonstrated that dominance affected facilitation and inhibition effects differently. There was more facilitation for high-dominance items than for low-dominance items $[F(1,60)=9.15, M S e=868]$. The facilitation effect for high-dominance items in the related condition was reliable $[F(1,60)=9.74, M S \mathrm{e}=903]$; the corresponding effect for low-dominance items was not reliable $(F<1)$. The effects of dominance on the facilitation effects were the same for lexical decision and naming $(F<1)$. In contrast to the pattern of facilitation effects, the inhibition effects did not depend upon the dominance of the target word $(F<1)$. As seen in Table 2 , inhibition effects within each task were of approximately the same magnitude for both high- and lowdominance targets.
The only other finding was that responses were faster in naming than in lexical decision $[F(1,60)=11.15, M S \mathrm{e}$ $=22,316$ for the main effect of task].

Errors. The error results are also displayed in Table 2. Again, the results were very similar to those of Experiment 1 . The pattern of priming effects differed for the two tasks $[F(2,120)=14.81, M S e=.0141$ for the task $\times$ prime interaction]. The basis of the difference was that inhibition effects were observed only in the unrelated condition of the lexical decision task $[F(1,18)=19.46, M S e$ $=.0113$.

In summary, the findings of Experiment 2 were very similar to those of Experiment 1 . In both experiments, large inhibition effects were found in lexical decision but not in naming. On the other hand, the facilitation effects for the two tasks were similar in magnitude in both experiments. Furthermore, dominance influenced the size of the facilitation effects similarly for naming and lexical decision in Experiment 2. These results suggest that facilitation effects in the two tasks are due to the same underlying mechanism, whereas inhibition effects are due to processes which are unique to the lexical decision task. Experiment 3 provides a further test of this hypothesis.

\section{EXPERIMENT 3}

The findings of Experiments 1 and 2 are consistent with Becker's (1980) finding that inhibition dominance results when category-exemplar pairs are used as stimuli in the lexical decision task. Becker also demonstrated that using free associates instead of category-exemplar pairs results in facilitation dominance in the lexical decision task. In Experiment 3, we used free associates to provide a further test of the locus of priming effects in lexical decision. If facilitation effects in naming and lexical decision are due to common prelexical processes, then the facilitation effects in Experiment 3 should increase in magnitude for both tasks, relative to Experiments 1 and 2. Inhibition effects should decrease in the lexical decision task if Becker's findings are replicated. However, inhibition effects in naming should not be affected by the change in items if inhibition is due to postlexical processes operating only in lexical decision.

A second purpose of Experiment 3 was to demonstrate that the failure to find inhibition of naming in Experiments 1 and 2 was not due to a lack of sensitivity of the task.

Table 2

Mean Reaction Times and Percentage Errors (in parentheses) as a Function of Task, Prime, and Dominance in Experiment 2

\begin{tabular}{llccccc}
\hline Task & Dom & Related & \multicolumn{1}{c}{ Neutral } & Unrelated & Facilitation & Inhibition \\
\hline Lexical Decision & High & $537(3.75)$ & $557(3.33)$ & $599(16.67)$ & $20(-0.42)$ & $42(13.34)$ \\
& Low & $573(9.38)$ & $567(10.00)$ & $619(27.50)$ & $-6(0.62)$ & $52(17.50)$ \\
\multirow{4}{*}{ Naming } & Mean & $555(6.56)$ & $562(6.67)$ & $609(22.09)$ & $7(0.11)$ & $47(15.42)$ \\
& High & $512(4.06)$ & $525(7.08)$ & $534(7.08)$ & $13(3.02)$ & $9(0.00)$ \\
& Low & $526(4.79)$ & $520(4.58)$ & $528(6.25)$ & $-6(-0.21)$ & $8(1.67)$ \\
& Mean & $519(4.43)$ & $523(5.83)$ & $531(6.67)$ & $4(1.40)$ & $8(0.84)$ \\
\hline
\end{tabular}


This argument might be made because the only reliable priming effect on naming demonstrated so far was the 13msec facilitation effect observed for high-dominance items in Experiment 2. If large facilitation effects are found in Experiment 3, the sensitivity of the naming procedure will be demonstrated.

\section{Method}

Subjects. Subjects were undergraduate students at the University of Kentucky who participated in partial fulfillment of a course requirement. Excluding 12 subjects whose error rates on target words exceeded $17 \%, 24$ subjects were assigned to the lexical decision task and 48 subjects were assigned to the naming task. Of the 12 excluded subjects, 1 was from the lexical decision condition and 11 were from the naming condition.

Materials. The critical stimuli were based upon a set of 60 associated word pairs. The word pairs represented a variety of semantic relations, but all pairs were strong associates (e.g., war-peace, letter-number, flower-rose, zebra-stripes). The second word in each pair served as the target word in all experimental conditions. The word pairs were divided at random into two blocks of 30 pairs each. Within each block, 20 related prime pairs were formed by using the first word of each associated pair as the prime and the second word as the target (e.g., war-peace). An additional 5 neutral prime pairs were constructed by replacing the first word of the original pair with the word blank. The other 5 items in each block were unrelated pairs constructed by replacing the first word of the original word pair with the 5 words that were replaced by blank in the neutral condition (e.g., flower-number). Six different lists of 60 pairs each were constructed to counterbalance the assignment of target words to priming conditions.

The generation of nonword items, buffer items, and practice items followed procedures analogous to those of Experiments 1 and 2.

Procedure. The procedure was the same as in Experiments 1 and 2.

\section{Results}

The design of the experiment included the betweensubjects factors of task and list and the within-subjects factor of prime.

Reaction times. The results of Experiment 3 are summarized in Table 3. As seen in the table, the effects of the priming manipulation depended upon the task $[F(2,120)=5.27, M S e=916$ for the task $\times$ prime interaction]. Subjects responded more quickly in the related prime condition than in the neutral prime condition, both in the lexical decision task $[F(1,18)=31.10, M S e=$ $1,340]$ and in the naming task $[F(1,42)=56.54, M S e$ =676]. Subjects in the lexical decision task responded more slowly in the unrelated prime condition than in the neutral condition $[F(1,18)=4.92, M S e=1,576]$. However, the inhibition effect was not reliable in the naming task $[F(1,42)=2.32, M S \mathrm{e}=986, p>.1]$. The only other reliable effect of interest was that responses were faster in naming than in lexical decision $[F(1,60)=16.81$, $M S e=8,552$ for the main effect of task].

In addition to the preceding analyses, four comparisons were made across Experiments 2 and 3 to determine whether the change from category-exemplars to free associates influenced the magnitude of the facilitation and inhibition effects as predicted. (Experiment 1 was not included in the comparisons because it involved the repeated presentation of a small set of prime words, unlike Experiments 2 and 3.) Facilitation effects were larger when free associates were used as stimuli in both the lexical decision task $[F(1,36)=28.41, M S e=1,100]$ and the naming task $[F(1,84)=56.91, M S e=577]$. Inhibition decreased when free associates were used in the lexical decision task $[F(1,36)=3.52, M S e=1,803, p<.05$, one tailed]. However, there was no difference in the magnitude of the inhibition effects on naming in Experiments 2 and $3(F<1)$.

Errors. Analyses conducted on the error data demonstrated no reliable effects.

In summary, using free associates instead of categoryexemplar pairs as stimuli affected the pattern of priming effects on lexical decision (Becker, 1980). Whereas inhibition dominance was found in Experiment 2, facilitation dominance was observed in Experiment 3. The change in items also resulted in a large facilitation effect in the naming task. This result is important because it demonstrates that the lack of inhibition effects in the task cannot be attributed to an insensitive procedure. Finally, the inhibition effect on naming was unaffected by the change in items; rather, it remained small and unreliable. Let us now consider the implications of the findings of the three experiments.

\section{DISCUSSION}

Following others (Balota \& Chumbley, 1984; Balota \& Lorch, in press; Chumbley \& Balota, 1984; Lupker, 1984; Seidenberg et al., 1984), we have argued that priming effects in the lexical decision task can represent effects on either prelexical or postlexical access processing. On the assumption that naming involves minimal postlexical access processing, the locus of priming effects in lexical decision can be determined by comparing priming effects for lexical decision and naming. Manipulations having similar effects in the two tasks are interpreted as having effects on the common prelexical processes of the two tasks; effects that occur only in lexical decision are attributed to postlexical processes. Based on this logic, the results of our experiments indicate two general conclu-

Table 3

Mean Reaction Times and Percentage Errors (in parentheses) as a Function of Task and Prime for Experiment 3

\begin{tabular}{lcccll}
\hline Task & Related & Neutral & Unrelated & Facilitation & Inhibition \\
\hline Lexical Decision & $518(4.48)$ & $577(6.25)$ & $603(8.75)$ & $59(1.77)$ & $26(2.50)$ \\
Naming & $481(7.60)$ & $521(5.21)$ & $531(6.88)$ & $40(-2.39)$ & $10(1.67)$ \\
\hline
\end{tabular}


sions: (1) at least under conditions comparable to those of the present experiments, the facilitatory effects of related primes are prelexical in locus; and (2) in contrast, the inhibitory effects of unrelated primes are postlexical.

Consider the effects of the related primes across the three experiments. The lexical decision and naming tasks showed very similar patterns of facilitation effects. Facilitation effects were of similar magnitudes for the two tasks in each experiment (across experiments, the facilitation effect was $24 \mathrm{msec}$ in lexical decision and $19 \mathrm{msec}$ in naming). In addition, more facilitation was found for highdominance exemplars than for low-dominance exemplars in both tasks of Experiment 2 (see also Becker, 1980, Experiment 5; Lorch, 1982). Again, the magnitude of the dominance effect was similar for lexical decision and naming. Finally, facilitation effects were larger in both tasks when free associates were used as stimuli (Experiment 3) than when category-exemplar pairs were used (Experiments 1 and 2). Other investigators have also noted similar facilitation effects in naming and lexical decision under conditions comparable to the present experiments (Seidenberg et al., 1984; West \& Stanovich, 1982). These results suggest that the observed facilitation effects were due to the same prelexical mechanism in both tasks.

The most important findings concern the pattern of inhibition effects. Whereas other investigators have demonstrated different priming effects for the lexical decision and naming tasks (e.g., Lupker, 1984; Seidenberg et al., 1984), this is the first demonstration that inhibition effects depend upon the task in a single-word priming procedure. These results deserve careful consideration.

\section{Interpretation of Inhibition Effects}

Several issues arise concerning the pattern of inhibition effects observed in these experiments. The question of primary concern is whether inhibition effects observed in the lexical decision task are pre- or postlexical in nature. Three related findings indicate that the inhibition effects in lexical decision are due to postlexical processing. First, the findings that facilitation effects behaved similarly in the two tasks whereas inhibition effects differed suggests that facilitation and inhibition effects in lexical decision are due to different processes. Second, inhibition effects were affected by the change in items from Experiment 2 to Experiment 3 in the lexical decision task but not in the naming task. This result indicates that the inhibition effects in lexical decision were due to processes that were not involved in naming. Most important, the consistent finding that inhibition occurred in lexical decision but not in naming also suggests that processes unique to lexical decision were responsible for the inhibition effects (across experiments, the inhibition effect was $38 \mathrm{msec}$ in lexical decision compared to $9 \mathrm{msec}$ in naming). We conclude that inhibition effects in lexical decision are due to postlexical processing rather than prelexical processing. Several potential objections to this conclusion must be considered, however.
It must be acknowledged that the consistent tendency toward inhibition in the naming task across the three experiments may indicate a real effect. Fortunately, as noted in the preceding paragraph, the conclusion that inhibition is postlexical in lexical decision does not rely solely on acceptance of the null hypothesis. In addition, the much larger inhibition effects in lexical decision than in naming suggest a prominent role for postlexical processes in the former task. Unfortunately, the issue of whether the inhibition effects in naming are real is particularly difficult to resolve because of the problem of selecting an appropriate baseline condition. The basline employed in the current experiments is probably a conservative one for assessing inhibition. It has been found that inhibition effects are approximately $10 \mathrm{msec}$ larger when the word blank is used as a neutral prime than when a row of Xs is used (de Groot et al., 1982). Thus, if a row of Xs had been used as the neutral prime (i.e., the neutral prime employed by Becker, 1980), the small inhibition effects observed in the naming task would be expected to disappear completely.

Although we failed to find convincing evidence of inhibition in naming in the current experiments, we do not wish to imply that inhibition cannot occur in naming. In fact, Becker (1982) observed inhibition of naming using sentence frames as primes for single-word targets. Other conditions might produce inhibition as well. For example, including nonwords in a naming task might encourage subjects to adopt a postlexical, binary decision strategy similar to that hypothesized for the lexical decision task. This might occur because the lexical status of a target item is relevant to the appropriate basis for deciding its pronunciation. Specifically, if a target is a nonword, then the subject might use grapheme-to-phoneme correspondence rules to name the target. However, if the target is a word, a correct pronunciation is assured only if the subject accesses the articulatory representation of the word (although a grapheme-to-phoneme translation may suffice most of the time). Regardless of whether inhibition effects can be produced in naming, the important point is that the inhibition effects in lexical decision that have been used to support Becker's model are not due to effects on word-recognition processes.

Are there alternative explanations of the finding that inhibition effects differed for lexical decision and naming? One possibility is that the two tasks involve entirely different processing strategies, as opposed to differing only with respect to postlexical processes. Perhaps subjects performed the naming task using grapheme-tophoneme translation rules for determining word pronunciations, whereas lexical decision responses were based on lexical access (Coltheart, Davelaar, Jonasson, \& Besner, 1977). However, this explanation cannot account for the large facilitation effect on naming in Experiment 3 or for the fact that the facilitation effect on naming in Experiment 2 depended upon the prime-target dominance. These findings demonstrate that lexical access was in- 
volved in the naming tasks of the present investigation. More generally, the similar patterns of facilitation effects found for lexical decision and naming support the assumption that the two tasks involve similar prelexical processes.

A final issue concerns the exact basis for the different inhibition effects in lexical decision and naming. We suggested in the introduction that inhibition effects in lexical decision may be due to the binary decision required in the procedure. Another potential basis for the different inhibition effects may be identified, however. Specifically, the lexical decision task included presentation of nonwords, whereas the naming task involved only word stimuli. It is important to note that determining which of these factors is responsible for the different pattern of inhibition effects in the two tasks is an issue distinct from the primary question of whether inhibition in lexical decision is pre- or postlexical. In fact, we are not able to distinguish which of these two factors is responsible for the dependence of the inhibition effects on the task. Although including nonwords in the naming conditions would have eliminated this confound, we were concerned that the inclusion of nonwords in the naming task might cause subjects to adopt processing strategies that would make it difficult to interpret the results of the condition. For example, there is some evidence that mixing words and nonwords in naming leads (Dutch) subjects to adopt strategies that avoid lexical access (Hudson \& Bergman, 1985). An alternative possibility is that including nonwords would have caused subjects to attend to the lexical status of target words. As suggested earlier, this might have resulted in the use of the type of postlexical processing strategy that we sought to minimize in using the naming task.

In summary, the difference in the magnitude of the inhibition effects for the naming and lexical decision tasks suggests that postlexical processes are responsible for inhibition effects in lexical decision. This conclusion has direct implications for Becker's (1980) verification model.

\section{Implications for the Verification Model}

The verification model states that subjects respond to a prime by preparing a set of associates to compare with the subsequent target in a top-down fashion. If the number of associates included in the semantic set is large, the top-down processing will delay comparison of the target with a set of lexical interpretations determined by sensory analysis. As a consequence, there will be little facilitation on related trials and large inhibition on unrelated trials. If the number of associates in the semantic set is small, facilitation dominance should be observed. Our findings for the lexical decision task are consistent with these predictions and with Becker's (1980) previous findings: Inhibition dominance resulted when categoryexemplar pairs were used as stimuli (Experiments 1 and 2), whereas facilitation dominance was found for free associates (Experiment 3 ). The findings for the naming task do not support the verification model, however. Given that lexical access is involved in the naming task, the verification model predicts that similar patterns of facilitation and inhibition dominance should have been observed for naming. The predicted inhibition effects were not found in the naming conditions of either experiment.

The verification model might be modified in either of at least two ways to account for the discrepant results of the lexical decision and naming tasks. One possibility is that the model is an accurate explanation of priming effects on decision processes in lexical decision, rather than an explanation of word-recognition processes. This proposal does not account for the similar facilitation effects found in lexical decision and naming, however. The second possibility retains the model's basic hypothesis that a prime influences lexical access. According to this modification, the process of comparing the semantic set is carried out in parallel with the process of comparing the sensory set with the target. Thus, facilitation is still attributed to finding a match in the semantic set before finding a match in the sensory set. Inhibition might be accounted for by assuming that the failure to locate a match in the semantic set biases the decision mechanism toward a negative response (for a similar argument, see Neely, 1977). This proposal explains the similar facilitation effects for lexical decision and naming by attributing the effects to the common lexical access processes of the two tasks. The differing patterns of inhibition are accounted for by the assumption that only the lexical decision task involves a decision stage of processing.

\section{Other Implications}

Although the present results and discussion have been presented within the framework of the verification model, the findings are also relevant to any theory attributing inhibition effects to processes operating prior to target presentation. Posner and Snyder's (1975a, 1975b) twoprocess theory is an important case in point. According to the theory, subjects generate expectations concerning a to-be-presented target if they are given sufficient time and motivation (i.e., high cue validity) to process a prime. Thus, in a semantic priming task, subjects will attend to that area of memory which is related to the prime word. If the target is related to the prime (i.e., is in the attended area of memory), response latency will be relatively fast because attention is already allocated to the appropriate area of memory. However, if the target is unrelated to the prime (i.e., in a different area of memory than that being attended), then response latency will be relatively slow because attention must be switched to the appropriate area of memory.

This explanation of inhibition effects is inadequate to account for the results of the present study. Because the priming conditions were the same in the lexical decision and naming tasks, similar patterns of inhibition should have been found in both tasks. The finding that inhibition effects were restricted to lexical decision is consistent with Posner and Snyder's (1975b) own suggestion 
that prime-target matching strategies may play an important role in binary choice tasks. As they note, such an interpretation is inconsistent with the hypothesis that inhibition effects are due to attentional processes operating prior to target presentation. Thus, the conclusion that inhibition effects are postlexical in the lexical decision task has serious implications for using the task to test Posner and Snyder's theory. This is because the presence or absence of inhibition effects is a primary empirical criterion for discriminating automatic and attentional processes (Balota, 1983; Neely, 1976, 1977).

\section{Conclusion}

In summary, the results of three experiments indicate that independent mechanisms are responsible for the facilitation and inhibition effects observed in lexical decision tasks. Most important, the inhibition effects appear to be due to postlexical processes involved in lexical decision. This conclusion has serious implications for any theory localizing inhibition effects on prelexical processes. In addition, it implies that investigators should carefully consider the appropriateness of the lexical decision task to their research problem. If the concern is to investigate lexical access or prelexical processes, an independent assessment of the locus of experimental effects should be considered if the lexical decision task is employed. Alternatively, the naming task might be used as a procedure that minimizes the role of postlexical processes.

\section{REFERENCES}

Balota, D. A. (1983). Automatic semantic activation and episodic memory encoding. Journal of Verbal Learning \& Verbal Behavior, 22, 88-104

Balota, D. A., \& Chumbley, J. I. (1984). Are lexical decisions a good measure of lexical access? The role of word frequency in the neglected decision stage. Journal of Experimental Psychology: Human Perception \& Performance, 10, 340-357.

Balota, D. A., \& LoRCH, R. F., JR. (in press). The depth of automatic spreading activation: Mediated priming effects in pronunciation but not in lexical decision. Journal of Experimental Psychology: Learning, Memory, \& Cognition.

Battig, W. F., \& Montague, W. E. (1969). Category norms for verbal items in 56 categories: A replication and extension of the Connecticut category norms. Journal of Experimental Psychology Monograph, 80(3, Pt. 2).

BECKER, C. A. (1980). Semantic context effects in visual word recognition: An analysis of semantic strategies. Memory \& Cognition, 8 , 493-512.

BECKER, C. A. (1982, November). Sentence context effects in children and adults. Paper presented at the meeting of the Psychonomic Society, Minneapolis.

BeCKER, C. A., \& KILLION, T. H. (1977). Interaction of visual and cognitive effects in word recognition. Journal of Experimental Psychology: Human Perception \& Performance, 3, 389-401.
Chumbley, J. I., \& Balota, D. A. (1984). A word's meaning affects the decision in lexical decision. Memory \& Cognition, 12, 590-606. Coltheart, M., Davelaar, E., Jonasson, J. T., \& Besner, D (1977). Access to the mental lexicon. In S. Dornic (Ed.), Attention and performance VI. Hillsdale, NJ: Erlbaum.

DE Groot, A. M. B. (1983). The range of automatic spreading activation in word priming. Journal of Verbal Learning \& Verbal Behavior, 22, 417-436.

de Groot, A. M. B., Thomassen, A. J. W. M., \& Hudson, P. T. W. (1982). Associative facilitation of word recognition as measured from a neutral prime. Memory \& Cognition, 10, 358-370.

den Heyer, K., Briand, K., \& Dannenbring, G. L. (1983). Strategic factors in a lexical-decision task: Evidence for automatic and attention-driven processes. Memory \& Cognition, 11, 374-381.

FORSTER, K. I. (1979). Levels of processing and the structure of the language processor. In W. E. Cooper \& E. Walker (Eds.), Sentence processing: Psycholinguistic studies presented to Merrill Garrett. Hillsdale, NJ: Erlbaum.

Hudson, P. T. W., \& Bergman, M. W. (1985). Lexical knowledge in word recognition: Word length and word frequency in naming and lexical decision tasks. Journal of Memory \& Language, 24, 46-58.

LORCH, R. F., JR. (1982). Priming and search processes in semantic memory: A test of three models of spreading activation. Journal of Verbal Learning \& Verbal Behavior, 21, 468-492.

LUPKER, S. J. (1984). Semantic priming without association: A second look. Journal of Verbal Learning \& Verbal Behavior, 23, 709-733.

Meyer, D. E., \& Schvaneveldt, R. W. (1971). Facilitation in recognizing pairs of words: Evidence of a dependence between retrieval operations. Journal of Experimental Psychology, 90, 227-234.

NeELY, J. H. (1976). Semantic priming and retrieval from lexical memory: Evidence for facilitatory and inhibitory processes. Memory \& Cognition, 4, 648-654.

NeELY, J. H. (1977). Semantic priming and retrieval from lexical memory: Roles of inhibitionless spreading activation and limitedcapacity attention. Journal of Experimental Psychology: General, 106, 226-254

PosNer, M. I., \& SNYDer, C. R. R. (1975a). Attention and cognitive control. In R. L. Solso (Ed.), Information processing and cognition: The Loyola Symposium. Hillsdale, NJ: Erlbaum.

Posner, M. I., \& SNyder, C. R. R. (1975b). Facilitation and inhibition in the processing of signals. In P. M. A. Rabbitt (Ed.), Attention and performance $V$. London: Academic Press.

Seidenberg, M. S., Waters, G. S., Sanders, M., \& Langer, P. (1984). Pre- and postlexical loci of contextual effects on word recognition. Memory \& Cognition, 12, 315-328.

Shapiro, S. I., \& Palermo, D. S. (1970). Conceptual organization and class membership: Normative data for representatives of 100 categories. Psychological Monograph Supplements, 3(11, Whole No. 43).

Theios, J., MUiSe, J. G. (1977). The word identification process in reading. In N. J. Castellan, D. B. Pisoni, \& G. R. Potts (Eds.), Cognitive theory (Vol. 2). Hillsdale, NJ: Erlbaum.

West, R. F. \& Stanovich, K. E. (1982). Source of inhibition in experiments on the effect of sentence context on word recognition. Journal of Experimental Psychology: Learning, Memory \& Cognition, 8 , 385-399.

(Manuscript received April 25, 1985; revision accepted for publication September 23, 1985.) 